
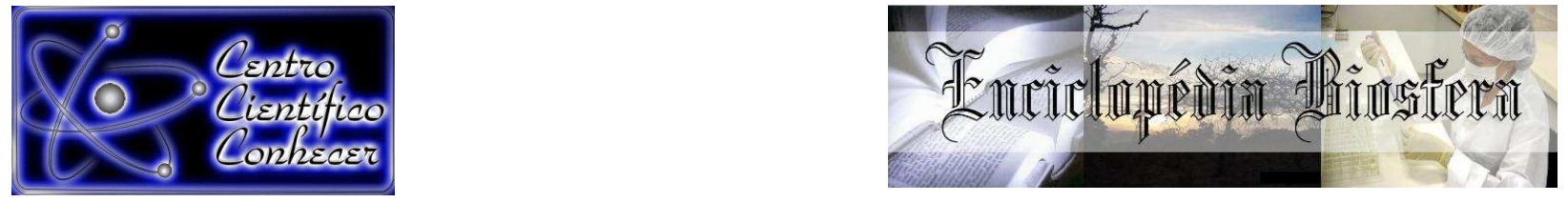

\title{
RESTRITORES HÍDRICOS E SEU USO NA INIBIÇÃO DA GERMINAÇÃO DE SEMENTES DE ABÓBORA E TOMATE DURANTE TESTE DE SANIDADE
}

Gabriele Fonseca de Paula ${ }^{1}$, Ewerton Gewehr ${ }^{2}$, Henrique Lopes Chagas ${ }^{1}$, Andréia da Silva Almeida ${ }^{3}$, Gustavo Fonseca Rodrigues ${ }^{1}$

1 Graduando da Faculdade de Agronomia Eliseu Maciel Universidade Federal de Pelotas

2 Doutorando em Ciência e Tecnologia de Sementes, UFPel, Brasil

*ewertongewehr@hotmail.com

3 Pós-doutoranda em Ciência e Tecnologia de Sementes, Universidade Federal de

Pelotas, Pelotas (UFPel), Brasil

Recebido em: 08/04/2016 - Aprovado em: 30/05/2016 - Publicado em: 20/06/2016

DOI: 10.18677/Enciclopedia_Biosfera_2016_104

Objetivou-se avaliar a possibilidade do uso de restritores hídricos que inibam a germinação de sementes de abóbora e tomate, e a influência na incidência de microrganismos nas amostras avaliadas no teste de incubação em substrato de papel. O experimento foi realizado no laboratório de Análise de Sementes da Universidade Federal de Pelotas-RS. As sementes utilizadas no experimento foram de abóbora e tomate. Os tratamentos foram compostos de restritores hídricos sendo utilizados os seguintes produtos como restritores: polietileno glicol 6000 (PEG 6000), manitol, cloreto de potássio $(\mathrm{KCl})$ e cloreto de sódio $(\mathrm{NaCl})$, nos potenciais hídricos de $0,-0,3,-0,6,-0,9,-1,2$ e -1,6 MPa, sendo usado como testemunha água destilada e congelamento em ultra-freezer. Foram realizados os seguintes testes: Incubação das sementes com restritores hídricos; Incubação das sementes em água; Incubação das sementes em água com congelamento; Teste de Germinação. Todos os restritores hídricos testados inibiram a germinação de sementes de tomate e abóbora de forma eficiente e em baixo potencial hídrico. A utilização de restritores hídricos utilizados se mostrou eficiente para detecção de fungos em tomate e os restritores polietilenoglicol 6000 (PEG 6000), cloreto de potássio (KCl) em sementes de abóbora não prejudicaram o desenvolvimento dos fungos estudados sendo considerado eficiente na detecção de fungos.

PALAVRAS-CHAVE: Cucurbita spp, Patologia, Solanum lycopersicum.

\section{WATER RESTRICTORS AND THEIR USE IN THE INHIBITION OF GERMINATION OF TOMATO AND PUMPKIN SEEDS DURING SANITY CHECK.}

\begin{abstract}
The objective was to evaluate the possibility of using water restrictors that inhibits the pumpkin seed germination and tomato, and its influence on the incidence of microorganisms in the samples evaluated in the incubation test paper substrate. The
\end{abstract}


experiment was conducted at the Seed Analysis Laboratory of the Universidade Federal de Pelotas-RS. The seeds used in the experiment were pumpkin and tomato. The treatments were composed of water restrictors are used as restrictors following products: polyethylene glycol 6000 (PEG 6000), mannitol, potassium chloride $(\mathrm{KCl})$ and sodium chloride $(\mathrm{NaCl})$ in water potential of $0,-0.3,-0.6,-0.9,-1.2$ and -1.6 MPa, being used as a witness distilled water and freeze at ultra-freezer. Was performed the following tests: incubation of the seeds with water restrictors; Incubating seeds in water; Incubating seeds in water to freezing; Germination test. All water restrictors tested inhibit the germination of tomato seeds and pumpkin efficiently and at low water potential. The use of water restrictors used will prove effective for detection of fungi of tomato and polyethylene glycol restrictors 6000 (PEG 6000), potassium chloride (KCl) pumpkin seeds did not hinder the development of fungi studied being considered effective in fungal detection.

KEYWORDS: Cucurbita spp, Patologia, Pathology, Solanum lycopersicum.

\section{INTRODUÇÃO}

Grande parte das informações sobre as condições sanitária de um lote de sementes provem de testes de patologia como "blotter test", este considerado de grande importância, pois fornece informações precisas as empresas produtoras de sementes, programas de certificação de sementes, e órgãos fiscalizadores entre outras. Além disso, auxilia os produtores e empresas a utilizarem de forma mais adequada o manejo de tratamento de sementes.

Um dos problemas enfrentados pelos laboratórios que realizam testes de patologia em sementes é a rápida germinação de algumas espécies durante o período de incubação do teste. Este problema pode prejudicar a avaliação do teste, o que acaba fornecendo resultados não confiáveis.

Dessa forma, o uso de restritores hídricos que contribuam para inibição da germinação das sementes durante o período de incubação dos testes sanitários, tem sido investigado por pesquisas científicas, principalmente para espécies de sementes de maior tamanho, principalmente soja, feijão e algodão. No entanto para espécies com sementes de tamanho menor, as pesquisas são escassas.

De acordo com o Manual de Análise Sanitária de Sementes (BRASIL, 2009), a inibição da germinação em sementes de espécies de dicotiledôneas, deve ser feita com o uso de solução de 2,4-D (2,4-diclorofenoxiacetato de sódio) a 5 ppm de concentração. Já para sementes de espécies de monocotiledôneas e dicotiledôneas com sementes menores, a técnica do congelamento pode ser utilizada em substituição ao 2,4D. Porém, o próprio manual cita que o uso de restritores hídricos pode ser uma alternativa ao uso de ambas formas de inibição de germinação, porém ainda necessita de pesquisa nessa área (SANCHES et al., 2015).

A técnica utilizando o 2,4-D possui alguns pontos negativos, pois o mesmo é um produto fungitóxico. Além disso, quando é usado em grandes concentrações, pode representar sérios riscos à saúde dos laboratoristas. Já a técnica de congelamento nos testes de sanidade, pode favorecer 0 aparecimento de microrganismos saprófitas (SANCHES et al., 2015), além de ser uma técnica com custos elevados, pois necessita de equipamentos com alto valor de compra, e a limitação na execução do teste.

A técnica de restrição pelo uso de solutos, tais como o manitol, polietilenoglicol (PEG), $\mathrm{KCl}$ e $\mathrm{NaCl}$, tem sido alvo de estudos visando inibir a 
germinação de sementes de diversas espécies, tais como feijão, soja, algodão, milho, trigo, etc. (JUNGES et al., 2014). Essa técnica consiste em embeber o papel filtro com restritores hídricos que permita o adequado controle da germinação das sementes, de maneira a não afetar o desenvolvimento de fungos associados a sementes (BEZERRA et al., 2013).

Os restritores hídricos inibem a germinação das sementes pelo efeito osmótico e/ou iônico que dificulta a absorção de água ou facilita a penetração de íons nas células. Esse efeito pode ser atribuído à menor mobilização das reservas, menor síntese e atividade enzimática e mudanças na turgência celular (BEZERRA et al., 2013).

Face ao exposto, objetivou-se avaliar a possibilidade do uso de restritores hídricos que inibam a germinação de sementes de abóbora e tomate, e a influência na incidência de microrganismos nas amostras avaliadas no teste de incubação em substrato de papel.

\section{MATERIAL E MÉTODOS}

O experimento foi realizado no laboratório de Análise de Sementes da UFPelPelotas-RS. As sementes utilizadas no experimento foram de abóbora da cultivar Goianinha e de tomate da cultivar Santa cruz. Os tratamentos foram compostos com restritores hídricos sendo utilizados os seguintes produtos: polietileno glicol 6000 (PEG 6000), manitol, cloreto de potássio (KCl) e cloreto de sódio ( $\mathrm{NaCl}$ ), nos potenciais hídricos de $0,-0,3,-0,6,-0,9,-1,2$ e -1,6 $\mathrm{MPa}$, sendo usado como testemunha água destilada e congelamento em ultra-freezer.

As soluções de manitol, $\mathrm{KCl}$ e $\mathrm{NaCl}$, foram preparadas segundo a fórmula proposta por VAN'T HOFF citado por SALISBURY \& ROSS, (1991): yos = -i RTC sendo: $y o s=$ potencial osmótico $(\mathrm{Mpa}) \mathrm{i}=$ Coeficiente isotônico, $\mathrm{R}=$ constante geral dos gases perfeitos $(0,0083 \mathrm{Mpa} \times 1 \times$ mol- $1 \times \mathrm{K}-1) \mathrm{T}=$ Temperatura absoluta $\left({ }^{\circ} \mathrm{K}\right) \mathrm{C}$ = concentração (mol/L). A solução de PEG 6000 foi preparada utilizando a tabela de potencial osmótico em função da concentração do polietileno glicol 6000 e da temperatura (VILLELA et al., 1991). Para obtenção dos restritores hídricos a quantidade de cada soluto foi calculada e depositada em frascos contendo $200 \mathrm{~mL}$ de água destilada, sendo estes em seguida, esterilizados em autoclave a $120^{\circ} \mathrm{C}$ por 20 minutos.

Incubação das sementes com restritores hídricos: utilizou-se como substrato duas folhas de papel filtro esterilizadas e umedecidas com os restritores hídricos, e acondicionadas em caixas plásticas do tipo "gerbox" $(11 \times 11 \times 3,5 \mathrm{~cm})$ desinfestadas com hipoclorito de sódio, logo após as sementes foram distribuídas, uniformemente, sobre o substrato de papel, em quatro repetições com 50 sementes cada, totalizando 200 sementes por tratamento. Posteriormente as caixas tipo gerbox contendo as sementes foram incubadas em câmara com $20 \pm 2{ }^{\circ} \mathrm{C}$ por sete dias em regime intermitente de $12 \mathrm{~h}$ de luz/12h de escuro.

Incubação das sementes em água: utilizou-se como substrato duas folhas de papel filtro esterilizadas e umedecidas com os restritores hídricos, e acondicionadas em caixas plásticas do tipo "gerbox" $(11 \times 11 \times 3,5 \mathrm{~cm})$ desinfestadas com hipoclorito de sódio, logo após as sementes foram distribuídas, uniformemente, sobre o substrato de papel, em quatro repetições com 50 sementes cada, totalizando 200 sementes por tratamento. Posteriormente as caixas tipo gerbox contendo as sementes foram incubadas a câmara com $20 \pm 2{ }^{\circ} \mathrm{C}$ por sete dias em regime intermitente de $12 \mathrm{~h}$ de luz/12h de escuro. 
Incubação das sementes em água com congelamento: utilizou-se como substrato duas folhas de papel filtro esterilizadas e umedecidas com os restritores hídricos, e acondicionadas em caixas plásticas do tipo "gerbox" $(11 \times 11 \times 3,5 \mathrm{~cm})$ desinfestadas com hipoclorito de sódio, logo após as sementes foram distribuídas, uniformemente, sobre o substrato de papel, em quatro repetições com 50 sementes cada, totalizando 200 sementes por tratamento. Logo após os gerbox foram incubados por 24 horas a uma temperatura de $20 \stackrel{\circ}{ } \mathrm{C}$, depois as mesmas foram colocadas em ultra-freezer a $-20{ }^{\circ} \mathrm{C}$ por 24 horas, posteriormente as caixas tipo gerbox contendo as sementes foram incubadas a câmara com $20 \pm 2{ }^{\circ} \mathrm{C}$ por sete dias em regime intermitente de $12 \mathrm{~h}$ de luz/12h de escuro.

A avaliação dos testes foi realizada com auxílio de microscópio estereoscópico, observando-se as estruturas dos patógenos, quando necessário utilizou-se também o microscópio biológico para identificação mais segura dos patógenos associados, segundo as Regras para Análise de Sementes (BRASIL,2009).

Teste de Germinação: foi realizado após o teste de sanidade, contabilizandose o número de plântulas germinadas em cada tratamento, utilizando o padrão de germinação botânica. Para o experimento foi utilizado delineamento inteiramente casualizado com quatro repetições para cada tratamento. Os dados foram submetidos à análise de variância, sendo os efeitos dos tratamentos avaliados pelo teste $F$, e quando significativo às médias foram comparas pelo teste de Tukey a $5 \%$ de probabilidade de erro. Os dados de percentagem de incidência de fungo foram transformados utilizando a formula $\sqrt{x+k}$.

\section{RESULTADOS E DISCUSSÃO}

Conforme a tabela 1 , verificou-se que para a cultura do tomate todos os restritores hídricos utilizados inibiram a germinação no menor potencial hídrico $(-0,3$ $\mathrm{MPa}$ ). Para a cultura da abóbora a inibição da germinação foi atingida no menor potencial hídrico $(-0,3 \mathrm{MPa})$ apenas nos restritores PEG (6000) e KCL, já os restritores $\mathrm{NaCL}$ e Manitol no menor potencial hídrico $(-0,3 \mathrm{MPa})$ ainda apresentaram baixos teores de germinação 9 e $8 \%$ respectivamente, sendo necessário a utilização de um potencial maior de -0,6 MPa para inibir totalmente a germinação. Resultados similares aos encontrados por ARAÚJO et el. (2012), que verificaram que a utilização de manitol, $\mathrm{NaCL}$ e $\mathrm{KCL}$, foram eficientes para inibir a germinação de sementes de algodão.

Com os resultados obtidos no experimento, pode-se afirmar que os restritores hídricos são uma alternativa de grande interesse, pois a utilização é de fácil manipulação e de baixo custo, quando comparado com a técnica de congelamento, sendo que a mesma apresenta a necessidades de utilização de ultra-freezer, equipamento com alto valor para aquisição.

Segundo CELANO et al. (2012), a técnica do congelamento pode danificar as células e promover o extravasamento de solutos das sementes para o substrato, favorecendo a contaminação secundária das sementes, podendo assim mascarar os resultados dos testes. Além disso, os mesmos autores relatam que os efeitos proporcionados pelos restritores inibem apenas a germinação, possibilitando um melhor resultado do teste de sanidade. 
TABELA 1: Valores de germinação (\%) de sementes de Abóbora e Tomate sob a influência de solutos e níveis de restrição hídrica em teste de sanidade substrato papel de filtro. UFPel, 2016.

\begin{tabular}{cccc}
\hline \multirow{2}{*}{ Tratamentos } & Nível de & \multicolumn{2}{c}{ Germinação (\%) } \\
\cline { 3 - 4 } & Restrição & Abóbora & Tomate \\
\hline Água & 0.0 & 75 & 32 \\
Congelamento & 0.0 & 0 & 0 \\
\hline \multirow{3}{*}{$\mathrm{NaCl}$} & -0.3 & 9 & 0 \\
& -0.6 & 0 & 0 \\
& -1.2 & 0 & 0 \\
$\mathrm{NEG}(6000)$ & -1.6 & 0 & 0 \\
\hline \multirow{3}{*}{$\mathrm{MANITOL}$} & -0.3 & 0 & 0 \\
& -0.6 & 0 & 0 \\
& -1.2 & 0 & 0 \\
& -1.6 & 0 & 0 \\
\hline & -0.3 & 8 & 0 \\
$\mathrm{KCl}$ & -0.6 & 0 & 0 \\
& -1.2 & 0 & 0 \\
& -1.6 & 0 & 0 \\
\hline & -0.3 & 0 & 0 \\
& -0.6 & 0 & 0 \\
& -1.2 & 0 & 0 \\
\hline
\end{tabular}

Através dos resultados obtidos pelo teste de germinação expostos na tabela 1 , selecionou-se para incubação em papel filtro "blotter test" na cultura do tomate apenas os restritores com nível de restrição de $-0,3 \mathrm{MPa}$ e para a cultura da abóbora os restritores selecionados foram, manitol e $\mathrm{NaCL}$ com nível de restrição de -0,6 e PEG6000 e KCL com potencial de restrição de -0,3 MPa. Essa seleção se deu, pois, os restritores com este potencial nas culturas estudadas foram capazes de inibir a germinação de forma eficiente.

Desse modo, pode-se observar na tabela 2, que para a detecção de Phoma sp., os restritores $\mathrm{NaCL}, \mathrm{KCL}$ e Manitol, foram eficientes na detecção do fungo, porém o $\mathrm{NaCL}$ foi o restritor que possibilitou verificação mais exata do fungo, não prejudicando e nem favorecendo o desenvolvimento do mesmo, fato este observado através da comparação com a incidência do fungo nos tratamentos sem restritores hídricos (água e a técnica de congelamento). Já o restritor PEG6000 prejudicou o desenvolvimento do fungo sendo considerado ineficiente para a detecção de Phoma sp.

Já para a detecção de Aspergillus sp. nenhum dos restritores hídricos detectaram de forma eficiente, pois quando comparado com a incidência de fungo nos métodos sem restrição hídrica, verificou-se que o mesmo favoreceu 0 desenvolvimento do fungo (Tabela 2).

De forma contraria os restritores manitol, PEG6000 e KCL se mostraram eficientes para a detecção da incidência de Penicillium sp., entretanto o manitol foi o restritor que teve melhor desempenho na detecção desse fungo, sendo que o valor 
em porcentagem do mesmo foi de $17 \%$, valor próximo ao encontrado em água e na técnica de congelamento, ambos com 13\% de incidência do fungo (Tabela 2).

TABELA 2: Incidência de fungos (\%) em sementes de tomate detectados em blotter test sem restrição hídrica e com restrição hídrica. UFPel, 2016.

\begin{tabular}{ccccc}
\hline \multirow{2}{*}{ Tratamentos } & Nível de & \multicolumn{3}{c}{ Incidência de fungos (\%) } \\
\cline { 3 - 5 } & Restrição & Phoma sp. & Aspergillus sp. & Penicillium sp. \\
\hline Água & 0 & $28 \mathrm{a}$ & $23 \mathrm{~cd}$ & $13 \mathrm{~b}$ \\
Congelamento & 0 & $27 \mathrm{a}$ & $16 \mathrm{~d}$ & $13 \mathrm{~b}$ \\
\hline NACL & -0.3 & $28 \mathrm{a}$ & $60 \mathrm{ab}$ & $36 \mathrm{a}$ \\
KCL & -0.3 & $20 \mathrm{ab}$ & $51 \mathrm{bc}$ & $31 \mathrm{ab}$ \\
MANITOL & -0.3 & $17 \mathrm{ab}$ & $60 \mathrm{abc}$ & $17 \mathrm{ab}$ \\
PEG & -0.3 & $8 \mathrm{~b}$ & $100 \mathrm{a}$ & $26 \mathrm{ab}$ \\
\hline CV $(\%)$ & & 18,04 & 17,45 & 18,95
\end{tabular}

Médias seguidas de mesma letra minúsculas na coluna não diferem significativamente entre si ao nível de $5 \%$ de probabilidade de erro pelo modelo de agrupamento de Tukey

$\mathrm{Na}$ tabela 3, verificou-se que para a detecção de Aspergillus sp., os restritores foram pouco eficientes, porem a incidência do fungo quando utilizado o KCL com potencial hídrico de $-0,3$ foi de $24 \%$ permitindo uma melhor verificação do fungo quando comparado com a incidência do mesmo em água e na técnica de congelamento.

$\mathrm{Na}$ avaliação de Rhizopus sp., o restritores NaCL e Manitol com potencial hídrico de -0,6 MPa, favoreceram o desenvolvimento do fungo quando comparado com a quantidade de fungo encontrado no teste sem restritores hídricos. Por outro lado, o restritor PEG6000 prejudicou o desenvolvimento de Rhizopus sp., a ponto de somente detectar $1 \%$ de incidência. Verificou-se que KCL com potencial de $-0,3 \mathrm{MPa}$ teve um bom desempenho para a detecção de Rhizopus sp., pois permitiu verificar a incidência de $17 \%$, valor próximo ao encontrado com os tratamentos sem restrição hídrica (Tabela 3).

Já para a detecção de Penicillium sp. os restritores PEG6000 e KCL com potencial hídrico de -0,3 MPa se mostraram eficazes, permitindo a detecção de 21 e $22 \%$ respectivamente, valores iguais aos encontrados nos tratamentos sem restrição hídrica. Entretanto, o manitol e o NaCL, se mostraram ineficazes na detecção de Penicillium sp., pois esses restritores promoveram o desenvolvimento do fungo (Tabela 3).

TABELA 3: Incidência de fungos (\%) em sementes de abóbora detectados em blotter test sem restrição hídrica e com restrição hídrica. UFPel, 2016.

\begin{tabular}{ccccc}
\hline \multirow{2}{*}{ Tratamentos } & Nível de & \multicolumn{3}{c}{ Incidência de fungos (\%) } \\
\cline { 2 - 5 } & Restrição & Aspergillus sp. & Rhizopus sp. & Penicillium sp. \\
\hline Água & 0 & $19 \mathrm{~cd}$ & $14 \mathrm{bc}$ & $34 \mathrm{~b}$ \\
Congelamento & 0 & $39 \mathrm{ab}$ & $4 \mathrm{c}$ & $22 \mathrm{~b}$ \\
\hline NACL & $-0,6$ & $24 \mathrm{cbd}$ & $17 \mathrm{~b}$ & $47 \mathrm{a}$ \\
KCL & $-0,3$ & $43 \mathrm{a}$ & $45 \mathrm{a}$ & $22 \mathrm{~b}$ \\
MANITOL & $-0,6$ & $32 \mathrm{ab}$ & $1 \mathrm{~d}$ & $21 \mathrm{~b}$ \\
PEG & $-0,3$ & 12,51 & 12,40 & 10,27 \\
\hline CV $(\%)$ & &
\end{tabular}

Médias seguidas de mesma letra minúsculas na coluna não diferem significativamente entre si em nível de 5\% de probabilidade de erro pelo modelo de agrupamento de Tukey 
Dessa forma, os resultados encontrados nessa pesquisa, possibilitam afirmar que os restritores hídricos podem ser utilizados como alternativa para inibir a germinação sem que afete na detecção de fungos nas sementes. Sendo que esses resultados confirmam os encontrados por CELANO et al. (2012), que relatam que o uso de restritores hídricos podem ser usados de forma segura para detecção de fungos em sementes de trigo. Além disso JúNIOR et al. (2008), descrevem que restritores hídricos como manitol, sacarose, cloreto de sódio e cloreto de potássio foram eficientes na detecção de Fusarium graminearum em trigo.

\section{CONCLUSÃO}

Todos os restritores hídricos testados inibiram a germinação de sementes de tomate e abóbora de forma eficiente e em baixo potencial hídrico.

A utilização de restritores hídricos utilizados se mostrou eficiente para detecção de fungos em tomate e os restritores polietilenoglicol 6000 (PEG 6000), cloreto de potássio $(\mathrm{KCl})$ em sementes de abóbora não prejudicou o desenvolvimento dos fungos estudados sendo considerado eficiente na detecção de fungos.

\section{REFERÊNCIAS}

ARAÚJO, A. E.; MENTEN, J. O. M.; DIAS, C. T. S.; CZERMAINSKI, A. B. C.; SANTOS, J. W.; MORAES, M. H. D. Efeito de restritores hídricos sobre a germinação, comprimento da radícula e níveis de detecção de Colletotrichum gossypii var. cephalosporioides em sementes de algodão. Summa phytopathol. v. 38, n. 1, p. 79-83, 2012. <http://dx.doi.org/10.1590/S0100-54052012000100013>. doi: 10.1590/S0100-54052012000100013.

BEZERRA, G. A.; MACEDO, D. A.; NASCIMENTO, I. O.; SOUSA, T. P.; COSTA, N. B.; SOUSA, L. F. R. A. Uso de bacillus spp. no controle de fitopatógenos em sementes de soja variedade BRS Valiosa-RR. Revista Agroecossistemas, v. 5, n. 1, p. 68-73, 2013.

BRASIL. Ministério da Agricultura, Pecuária e Abastecimento. Regras para análise de sementes. Ministério da Agricultura, Pecuária e Abastecimento. Secretaria de Defesa Agropecuária. Brasília, DF: Mapa/ACS, 395p. 2009.

CELANO, M. M.; MACHADO, J.C.; JACCOUD FILHO, D.S.; GUIMARÃES, R.M. Avaliação do potencial de uso da restrição hídrica em teste de sanidade de sementes de trigo visando à detecção de fungos. Revista Brasileira de Sementes, v. $34, \quad$ n. 4 p. 613 - 618, 2012. <http://dx.doi.org/10.1590/S010131222012000400012>. doi: 10.1590/S0101-31222012000400012.

JUNGES, E.; BASTOS, B. O.; TOEBE, M.; MULLER, J.; PEDROSO, D. C.; MUNIZ, M. F. B. Water restriction and seed coating in the microbiolization of maize seeds with Trichoderma spp. Comunicata Scientiae. v.5, n.1, p.18-25, 2014.

JÚNIOR, D. G.; VECHIATO, M. H.; MENTEN, J. O. M. Comparação de métodos para a detecção de Fusarium graminearum em sementes de trigo (Triticum aestivum 
L.). Summa Phytopathol, v. 34, n. 2, p. 164-167, 2008. <http://dx.doi.org/10.1590/S0100-54052008000200010>. doi: $\quad 10.1590 /$ S010054052008000200010 .

SANCHES, A. G.; MOREIRA, E. G. S.; COSTA, J. M.; SILVA, M. B.; CORDEIRO, C. A. M. Germinação e sanidade de sementes de cupuaçu (Theobroma grandiflorum) tratadas com fungincidas. Universidade Federal do Pará, Campus Altamira-Pará. HOLOS, v. 8, n. 31, p. 87-97, 2015. DOI: 10.15628/holos.2015.3825.

VILLELA, F.A., DONI-FILHO, L. SEQUEIRA, E.L. Tabela de potencial osmótico em função da concentração do polietileno glicol 6000 e da temperatura. Pesquisa Agropecuária Brasileira, v.26, n.11-12, p.1957-1968, 1991. 